Fermi National Accelerator Laboratory

FERMILAB-Conf-93/009-E

CDF

\title{
Sparticle Searches at CDF
}

\author{
Ping $\mathrm{Hu}$ \\ For the CDF Collaboration \\ Fermi National Accelerator Laboratory \\ P.O. Box 500, Batavia, Illinois 60510
}

January 1993

Published Proceedings Workshop on Ten Years of SUSY Confronting Experiment, CERN, Geneva, Switzerland, September 7-9, 1992 


\section{Disclaimer}

This report was prepared as an account of work sponsored by an agency of the United States Government. Neither the United States Government nor any agency thereof, nor any of their employees, makes any warranty, express or implied, or assumes any legal liability or responsibility for the accuracy, completeness, or usefulness of any information, apparatus, product, or process disclosed, or represents that its use would not infringe privately owned rights. Reference herein to any specific commercial product, process, or service by trade name, trademark, manufacturer, or otherwise, does not necessarily constitute or imply its endorsement, recommendation, or favoring by the United States Government or any agency thereof. The views and opinions of authors expressed herein do not necessarily state or reflect those of the United States Government or any agency thereof. 


\title{
Sparticle Searches at CDF
}

\author{
Ping $\mathrm{Hu}$ \\ CDF Collaboration
}

December 1992

\begin{abstract}
A set of jet events with large amount of Missing $E_{T}\left(W_{T}\right)$ from the Fermilab CDF experiment was analysed to search for SUSY signature. The absence of the signal resulted in squark and gluino mass limits $\left(m_{\tilde{q}}>126 \mathrm{GeV} / \mathrm{c}^{2}\right.$ and $\left.m_{\tilde{g}}>141 \mathrm{GeV} / \mathrm{c}^{2}\right)$. Some effects of some SUSY parameters on the squark and gluino mass limits are presented.
\end{abstract}

\section{Contents}

1 Introduction

2 The Minimal Supersymmetric Standard Model and Some Early Results

3 The Missing $E_{\mathrm{T}}$ Triggers 3

4 SUSY Event Selections 3

5 The Standard Model Background 5

6 Squark and Gluino Mass Limits 6

$\begin{array}{lll}7 & \text { Future Prospects } & 7\end{array}$ 


\section{Introduction}

In the supersymmetry (SUSY) theory [1], all fermions and bosons have partners with the same fundamental properties except spin and mass. The searches for such particles have conducted by a lot of experiments and various limits on SUSY particles' masses were established. The Fermilab Tevatron proton-antiproton collider operating at a center-of-mass energy of $1.8 \mathrm{TeV}$ provides an unique opportunity for SUSY search.

The Collider Detector at Fermilab (CDF) [2] is a large detector for study of $\vec{p} p$ collisions at the Fermilab Tevatron. It has a fine-grained, projectivetower geometry covering most of the $4 \pi$ solid angle with electromagnetic and hadron calorimeters. Its principal subsystems are the central scintillator sampling calorimeter $(|\eta|<1.1)$, the end-plug gas sampling calorimeter $(1.1<|\eta|<2.4)$, and the forward gas sampling calorimeter $(2.4<|\eta|<4.2)$, where the pseudorapidity $\eta=-\ln \tan \theta / 2$, and $\theta$ is the polar angle. Inside the central calorimeter, a superconducting solenoid generates a $1.41 \mathrm{~T}$ mag. netic field for tracking chambers surrounding the collision axis. The region $|\eta|<0.63$ is instrumented with drift chambers for muon detection outside of the hadron calorimeter. Charged tracks with $|\eta|>0.63$ associated with minimum ionization signals in the calorimeters are also considered muon candidates.

\section{The Minimal Supersymmetric Standard Model and Some Early Results}

In the Minimal Supersymmetric Standard Model (MSSM), the gauginos are complex mixtures of the higgsino, photino, zino and wino. Their masses are determined by five free parameters, which can be chosen as a SUSY Higgsino mass mixing parameter $\mu$, the ratio of the two Higgs vacuum expectation values $\tan \beta$, and the masses of the charged Higgs, squark and gluino $\left(m_{H^{+}}\right.$, $m_{q}$ and $\left.m_{\delta}\right)$. Most of the theoretical models also assume $R$-parity is conserved. This implies that SUSY particles are pair produced and that the lightest supersymmetric particle (LSP) cannot decay.

Gluinos and squarks are strongly interacting, thus they would be the SUSY particles with the largest production cross-sections at a $\bar{p} p$ collider. Fig. 1 show the expected $\tilde{q} \tilde{q}, \tilde{g} \tilde{g}$ and $\tilde{q} \tilde{g}$ cross sections for different squark 
and gluino masses. The branching ratios of gluino and squark decaying into various chargino and neutralino states depend on the the respective masses and mixing angles. In a scenario of a low mass gluino and a massless photino $(\tilde{\gamma})$ as the LSP, the decay modes become very simple. If $m_{\tilde{g}}<m_{\tilde{q}}$, the squark decays dominantly into $\bar{g} q$ and the main gluino decay is $\tilde{g} \rightarrow q \bar{q} \bar{\gamma}$. If $m_{\bar{q}}<m_{\bar{g}}$, then the gluino decays dominantly into $\bar{q} \bar{q}$ and the main squark decay is $\tilde{q} \rightarrow q \bar{\gamma}$. Since the photinos escape the detectors without any signature, the SUSY events would have two or more jets with a large amount of imbalanced transverse momenta.

UA1 and UA2 have conducted these searches at CERN $S \bar{p} p S$ collider and established squark and gluino mass limits [3]. CDF did a similar search with a $25 \mathrm{nb}^{-1}$ data set in 1987 . In the analysis, events with significant $\mathbb{E}_{\mathrm{T}}$ and a leading jet $E_{\mathrm{T}}>15 \mathrm{GeV}$ were selected. None of the events had $\mathbb{E}_{\mathrm{T}}$ greater then $40 \mathrm{GeV}$. This yielded a limit of $m_{q}>74 \mathrm{GeV} / \mathrm{c}^{2}$ and $m_{\S}>73 \mathrm{GeV} / \mathrm{c}^{2}$ at $90 \% \mathrm{CL}$ [4].

\section{The Missing $\mathrm{E}_{\mathrm{T}}$ Triggers}

The Level 2 and Level $3 \mathbb{Z}_{\mathrm{T}}$ triggers were installed in 1988-1989. The Level $2 E_{\mathrm{T}}$ trigger requires events to have

1. $\not Z_{\mathrm{T}}>25 \mathrm{GeV}$;

2. the leading calorimeter cluster has greater than $6 \mathrm{GeV}$ energy deposited in EM calorimeters and no seed towers in the forward region.

Fig. 2 shows the Level 2 trigger efficiency based on a study from a jet data sample. For $\mathbb{E}_{\mathbf{x}}>40 \mathrm{GeV}$, it is over $90 \%$ efficient.

The Level 3 trigger algorithm removes the detector noises and corrects the 'Texas Tower'. A dijet cut is used to reduced the QCD background. The dijet events are those having a jet $\left(E_{\mathrm{T}}>15 \mathrm{GeV}\right)$ within $30^{\circ}$ opposite to the leading jet direction in $\phi$. A cut of $\mathbb{E}_{\mathrm{T}}>40 \mathrm{GeV}$ is applied to those events.

\section{SUSY Event Selections}

The offline analysis eliminates known sources of detector noise, computes tower energies, reconstructs tracks, and applies the CDF jet algorithm which 
sums the calorimeter $\mathrm{E}_{\mathrm{x}}$ within a cone of 0.7 in $\eta-\phi$ space [5]. Events with cosmic rays are rejected. The dijet events (as discussed in the previous section) are also removed from the data sample. Events are selected by requiring $\mathbb{E}_{\mathrm{T}} \geq 20 \mathrm{GeV}$ and $\geq 2$ jets, where the jets are in the interval $|\eta|<$ 3.5 , have $\mathrm{E}_{\mathrm{T}} \geq 15 \mathrm{GeV}$, and deposit between $10 \%$ and $90 \%$ of their energy in the EM calorimeters. This selection yields 1,226 events.

A series of more stringent cuts are made in order to get a final sample of events which could contain SUSY particles. The first of these cuts is designed to select events with a well measured large $\mathbb{Z}_{\mathrm{T}}$ by requiring $\mathbb{E}_{\mathrm{T}} \geq 40 \mathrm{GeV}(281$ events survive), and $\mathbb{E}_{\mathrm{T}}$ significance $S \geq 2.8$, where $S \equiv \mathbb{E}_{\mathrm{T}} / \sqrt{\Sigma \mathrm{E}_{\mathrm{T}}}\left[\mathrm{GeV}^{1 / 2}\right]$ and the sum is over all calorimeter cells (257 events survive). The $S$ cut removes most events with $\mathbb{E}_{\mathrm{r}}$ induced by measurement fluctuations. For an event sample with no muons, neutrinos or other non-interacting particles, we expect the $S$ distribution to reflect the $\mathbb{E}_{\mathrm{T}}$ resolution of the detector. Fig. 3 shows the observed $S$ distribution for dijet events (jet $\mathrm{E}_{\mathrm{r}}>25 \mathrm{GeV}$ ) to be adequately described by the CDF detector simulation program. This gives confidence that, for events with jets in this $\mathrm{E}_{\mathrm{T}}$ range, the simulation correctly models the detector resolution.

We next required:

1. No muon candidates of transverse momentum $P_{T}>15 \mathrm{GeV} / \mathrm{c}$. This rejects $W \rightarrow \mu \nu$ and $Z \rightarrow \mu^{+} \mu^{-}$decays (230 events survive).

2. No calorimeter clusters with $E_{\mathrm{T}}>15 \mathrm{GeV}$ and $\geq 90 \%$ energy deposited in EM calorimeters. This rejects $W \rightarrow e \nu$ decays (196 events survive).

3. No jet cluster within $\pm 30^{\circ}$ in $\phi$ from the $\mathbb{E}_{\mathrm{T}}$ direction. This rejects mismeasured multijet events (124 events survive).

4. At least one central jet $(|\eta|<1.0)$ with a ratio of summed chargedtrack momenta to cluster energy $\geq 0.2$. This rejects events where timing information from the central hadron calorimeter was unavailable to eliminate cosmic rays (116 events survive).

5. An interaction vertex within $\pm 60 \mathrm{~cm}$ of the detector center on the beam axis and no other beam interaction vertex (100 events survive).

Remaining events are inspected on a graphics display. We remove one beam-gas collision, one cosmic ray event, and five events with detector mal- 
functions. The final sample of 93 events has 71 events with two jets, 20 with three jets and 2 with four jets (jet $\mathrm{E}_{\mathbf{T}}>15 \mathrm{GeV}$ ).

\section{The Standard Model Background}

The main sources of non-SUSY background in the resulting $E_{\mathrm{r}}$ plus jets data sample are from QCD multijet events in which the jet energy has been mismeasured, and events associated with $W / Z$ production and leptonic decay. For $W / Z$ plus jet events, if the lepton's $P_{T}$ is below $15 \mathrm{GeV} / \mathrm{c}$ or it is out of the detector geometric coverage, it could become the SUSY background. Mismeasured QCD events could become background since it resulted in the imbalanced $E_{T}$. Fig. 4 shows the angular separation between the third jet and the $\mathbb{E}_{\mathrm{T}}$ direction in $\phi$ before the cut 3 above applied. There are an overflow near $0^{\circ}$ while the same distributions for SUSY signal and W/Z plus jet events are almost flat. This is a clear indication that one of the jet energy is mismeasured which resulted in a large amount of $Z_{\mathrm{T}}$ in these event. The same distribution after cut 3 shows much less overflow near $0^{\circ}$. It is estimated that $4 \pm 4$ QCD events still remain after the cut.

Backgrounds from $W$ and $Z$ production and decay which pass our selection cuts are calculated with a Monte Carlo program [7] and a simulation of our detector. This predicts $23 \pm 8 Z \rightarrow \nu \bar{\nu}, 41 \pm 15 W \rightarrow \tau \nu, 18 \pm 6 W \rightarrow \mu \nu$, and $9 \pm 3 W \rightarrow e \nu$ events in our data sample. The total predicted event rate from background ( $95 \pm 19$ events) and its associated $\mathbb{E}_{r}$ spectrum agree well with the rate and spectrum for the 93 events in our data (Fig. 5).

We observe two events with $\mathbb{E}_{\mathrm{T}}>150 \mathrm{GeV}$. The highest $\mathbb{E}_{\mathrm{T}}$ event has $E_{\mathrm{T}}=185.9 \mathrm{GeV}$ with three jet clusters: $\mathrm{E}_{\mathrm{T}}=183.9,33.8$ and $11.3 \mathrm{GeV}$. The second highest $\mathbb{E}_{\mathrm{T}}$ event has $\mathbb{Z}_{\mathrm{T}}=167.8 \mathrm{GeV}$ with four jets: $E_{\mathrm{T}}=144.7,46.6$, 19.3 and $16.5 \mathrm{GeV}$. The third of these jets contains an electron candidate with $P_{T}=11.3 \mathrm{GeV} / \mathrm{c}$. The transverse mass calculated from the electron and $W_{T}$ vector is $57.2 \mathrm{GeV} / \mathrm{c}^{2}$. The $\mathrm{W} / \mathrm{Z}$ plus jets Monte Carlo calculation predicts 0.2 events with $E_{\mathrm{T}}>150 \mathrm{GeV}$ will pass our cuts. However note that this Monte Carlo program $\mathrm{W} / \mathrm{Z}$ production with up to three jets. There could be background from top events, too. At this time, we do not consider it since the top quark is still to be discovered. We believe that the two observed events do not constitute a statistically significant deviation from the standard model prediction. 


\section{Squark and Gluino Mass Limits}

To explore our sensitivity to a SUSY signal, we generate SUSY events using the ISAJET [8] Monte Carlo program (version 6.22) and EHLQ1, EHLQ2, DO1, and DO2 structure functions. The lowest rate comes from EHLQ1, which is used to provide a conservative production limit for SUSY particles. There are several sources of uncertainty in the predicted rate: $\pm 6.8 \%$ in rate from the the integrated luminosity, $\pm 10 \%$ in rate from the $\pm 5 \%$ uncertainty in the energy scale, $\pm 3 \%$ in rate from the uncertainty on the $\mathbb{Z}_{\mathrm{T}}$ trigger efficiency, and $\pm 15 \%$ from various sources in the Monte Carlo calculation the choice of $Q^{2}, \alpha_{0}$ evolution and the limited number of events generated. The combined acceptance of the simulated detector and analysis programs for generated SUSY events is heavily dependent on the choice of squark and gluino masses. For the mass region we studied, it varies from $3 \%$ to $25 \%$.

Our limits on $m_{\tilde{q}}$ and $m_{\tilde{g}}$ are based on a comparison of the observed $E_{\mathrm{T}}$ distribution with predictions for the standard model background based on the Monte Carlo of Ref. [7] and the estimated QCD background discussed above, plus the SUSY contribution based on the ISAJET Monte Carlo samples. For each hypothesised $m_{\tilde{q}}$ and $m_{\hat{q}}$ we fit the observed $\mathbb{E}_{\mathrm{T}}$ distribution over the full $\mathbb{E}_{\mathrm{T}}$ range using a binned likelihood method. The resulting upper limit on the rate of SUSY particle production is then compared with the predicted SUSY cross-section. Note that if the measured calorimeter energy scale is less than the true scale the predicted standard model contributions are reduced, and the limits are weakened. In extracting our limits to take into account this systematic uncertainty we have reduced the detector energy scale in the Monte Carlo simulation by $5 \%$. The resulting region of the $m_{q}$ vs. $m_{\text {更 }}$ plane excluded at $90 \%$ C.L. is shown in Fig. 6 . The symmetric and asymptotic points on the limiting boundary are: $m_{\tilde{q}}=m_{\tilde{g}}=m=225$

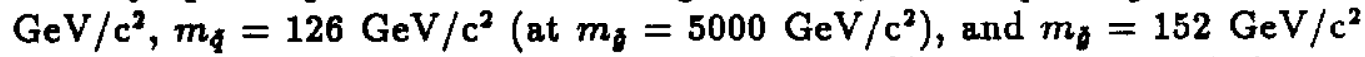
(at $m_{q}=5000 \mathrm{GeV} / \mathrm{c}^{2}$ ). We exclude at the $90 \%$ confidence level the existence of squarks and gluinos with masses less than $126 \mathrm{GeV} / \mathrm{c}^{2}$ and 141 $\mathrm{GeV} / \mathrm{c}^{2}$ respectively.

Finally we extracted the limits shown in Fig. 7 for cascade decays with a particular choice of SUSY parameters: $\mu=-250 \mathrm{GeV}, \tan \beta=2$, and $m_{H}$ $=500 \mathrm{GeV} / \mathrm{c}^{2}$ as used in Ref. [9]. The weakened limits are due to cascade decays and non-zero LSP mass. For a gluino mass greater than $410 \mathrm{GeV} / \mathrm{c}^{2}$, we can place no limit on the squark mass. It is worth to note that with this 
parameter set we choose, the degraded gluino mass limit $m_{\tilde{g}}>90 \mathrm{GeV} / \mathrm{c}^{2}$ (when $m_{\tilde{q}}$ is large) is the result of the cascade decay where the branching ratio for $\bar{g} \rightarrow q \bar{q} \bar{\chi}_{1}^{0}$ is only $34 \%$; but when $m_{\tilde{g}}$ is large the branching ratio for $\tilde{q} \rightarrow q \bar{\chi}_{1}^{0}$ is still about $100 \%$, the reduced squark mass limit is the result of the large LSP mass $\left(m_{\tilde{\chi}_{1}^{0}}=53 \mathrm{GeV} / \mathrm{c}^{2}\right.$ when $m_{\dot{g}}=400 \mathrm{GeV} / \mathrm{c}^{2}$ and $m_{\tilde{q}}=$ $\left.100 \mathrm{GeV} / \mathrm{c}^{2}\right)$.

\section{Future Prospects}

As the squark and gluino mass limits increases, the simple model with $m_{*}=0$ and no cascade is no longer valid. In MSSM there are five free parameters, we need a comprehensive method to study and present the limits on sparticle masses or other parameters. Such a study is on going. There is also the need to understand the background from $W W, W Z$ and $Z Z$ processes, as they will become important for high mass gluino and squarks.

The CDF has upgraded its detectors for the new run starting from 1992. The major improvements include the silicon-vertex detector which could provide information to tag $b$ jet, the central muon upgrade and extension system which could improve muon identification and muon coverage.

We expect to collect $100 \mathrm{pb}^{-1}$ data from this run. By that time, we should be able to probe the region with $m_{\tilde{g}}$ and $m_{\tilde{q}}$ up to $200 \mathrm{GeV} / \mathrm{c}^{2}$ and up to $300 \mathrm{GeV} / \mathrm{c}^{2}$ for $m_{\mathfrak{g}}=m_{\dot{q}}$.

\section{References}

[1] H. E. Haber and G. L. Kane, Phys. Rep. 117, 75 (1985); S. Dawson, E. Eichten and C. Quigg, Phys. Rev. D31, 1581 (1985).

[2] CDF Collaboration, F. Abe et al., Nucl. Instr. and Meth. A271, 387 (1988).

[3] UA1 Collaboration, C. Albajar et al., Phys. Lett. B198, 261 (1987); UA2 Collaboration, Phys. Lett. B235, 363 (1990).

[4] CDF Collaboration, F. Abe et al., Phys. Rev. Lett. 62, 1825 (1989).

[5] CDF Collaboration, F. Abe et al., Phys. Rev. D45, 1448 (1992). 


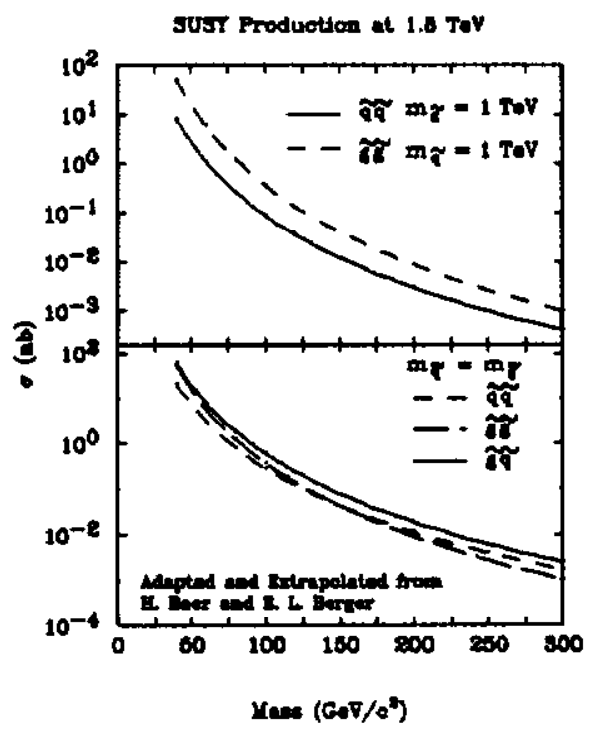

Figure 1: The squark and gluino production cross section at Tevatron. 


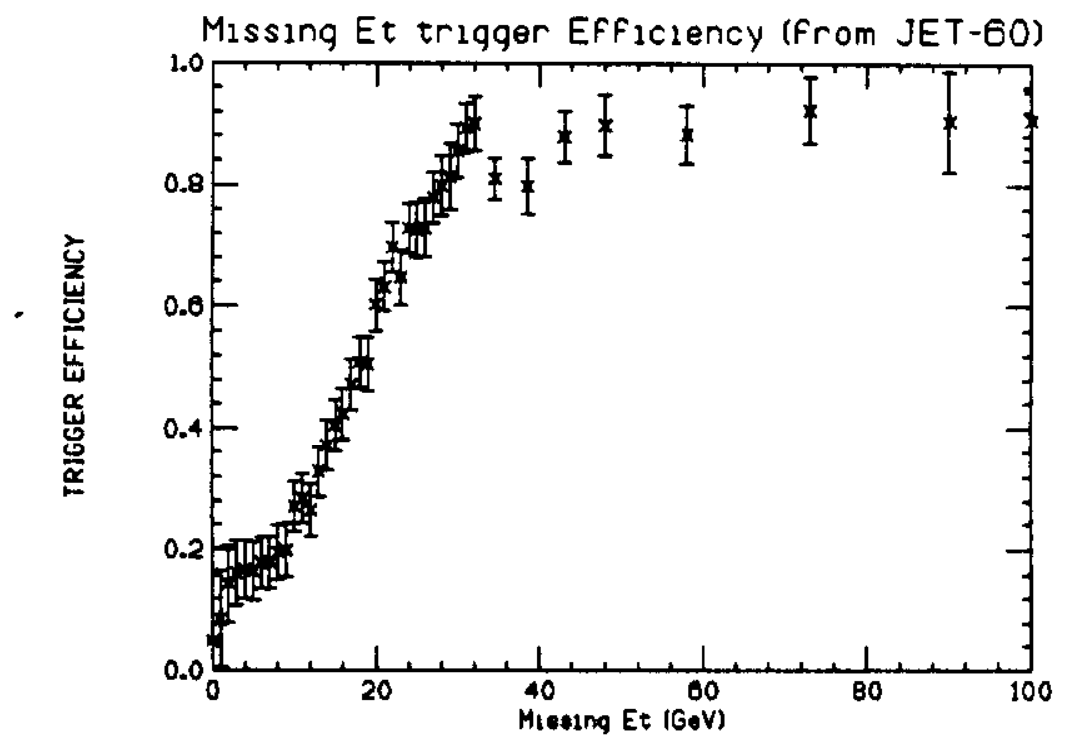

Figure 2: The Level $2 \mathbb{E}_{\mathrm{x}}$ trigger efficiency obtained from a jet date sample. 


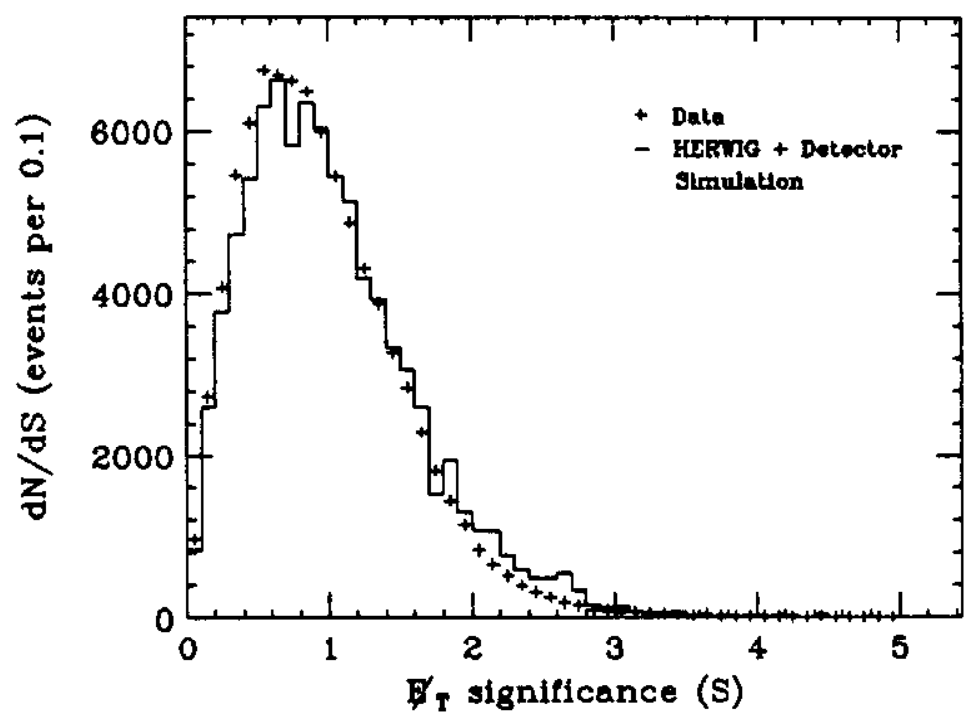

Figure 3: Missing $E_{\mathrm{r}}$ significance distribution for a jet sample (jet $E_{\mathrm{r}}>25$ $\mathrm{GeV}$ ) compared with the predictions from the HERWIG [6] Monte Carlo and $\mathrm{CDF}$ detector simulation. 


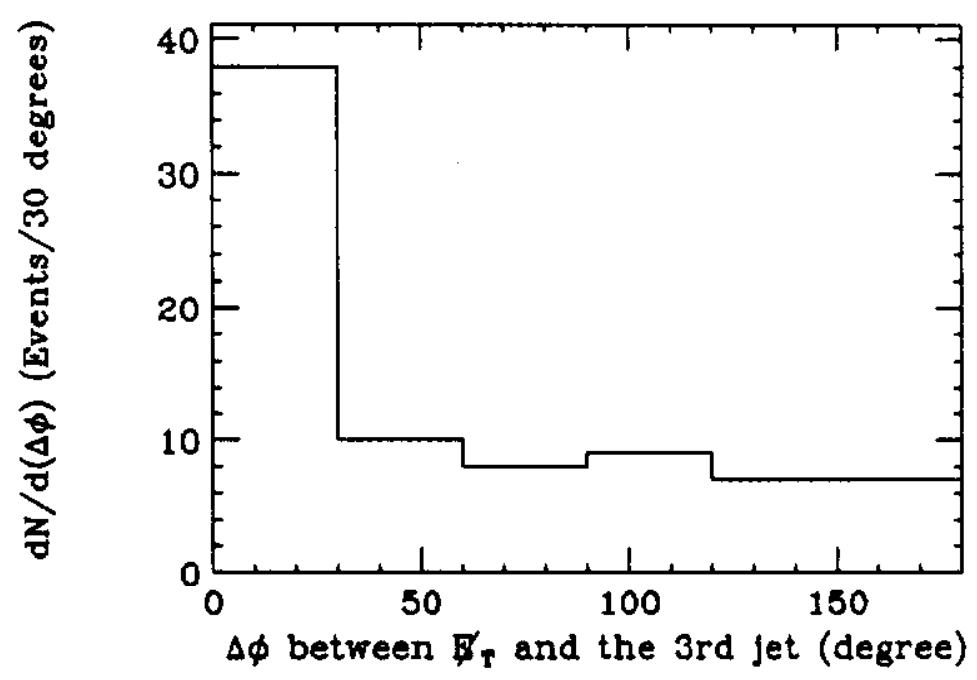

Figure 4: The $\phi$ angular seperation between $Z_{\mathrm{T}}$ and the third highest $E_{\mathrm{r}}$ jet. 


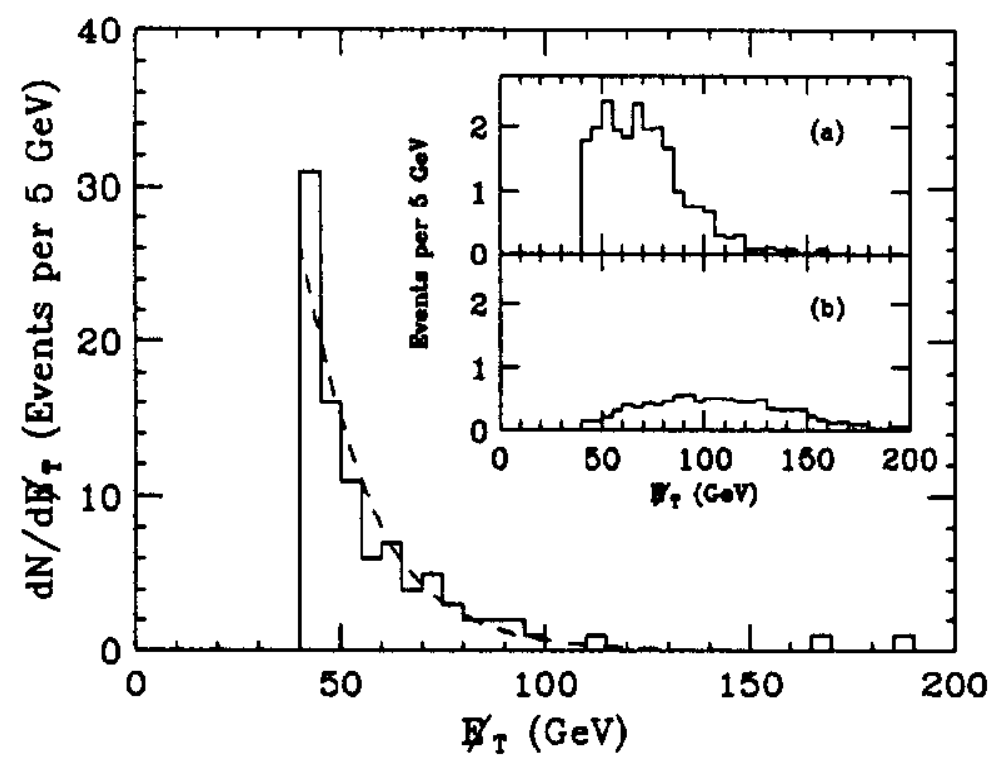

Figure 5: Missing $E_{T}$ distribution (solid line) for the data set described in the text, compared with the estimated background predictions (dashed line) obtained using the Monte Carlo program of reference [7] together with the CDF detector simulation plus the estimated QCD background. Insets show predicted $\vec{E}_{\mathrm{T}}$ distributions for squark and gluino production from ISAJET (version 6.22) and the CDF detector simulation for (a) $m_{\S}=125 \mathrm{GeV} / \mathrm{c}^{2}$ and $m_{\mathfrak{g}}=5000 \mathrm{GeV} / \mathrm{c}^{2}$, and $(\mathrm{b}) m_{\tilde{g}}=225 \mathrm{GeV} / \mathrm{c}^{2}$ and $m_{\tilde{q}}=225 \mathrm{GeV} / \mathrm{c}^{2}$. 


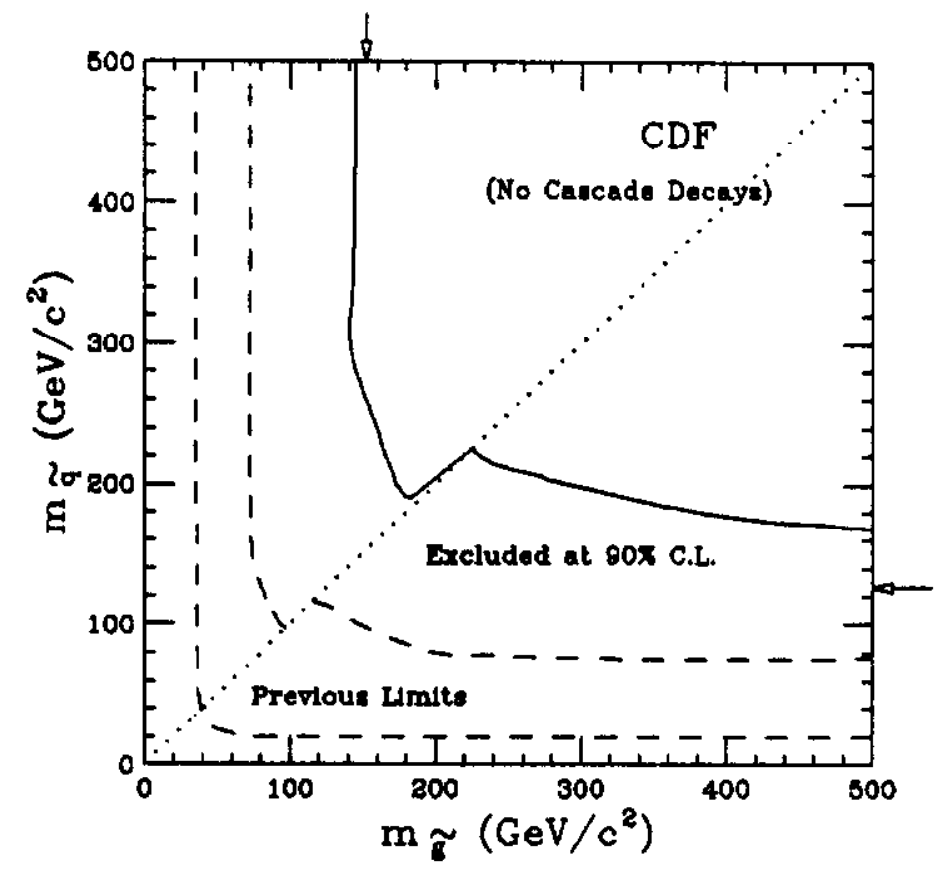

Figure 6: Squark and gluino mass limits for a version of SUSY with a light photino $\left(m_{\bar{q}}<15 \mathrm{GeV} / \mathrm{c}^{2}\right)$, six mass-degenerate squarks and no cascade decays. The region of $m_{q}$ versus $m_{g}$ plane excluded at $90 \%$ C.L. is shown. The dashed lines are boundaries of the region excluded by our previous analysis [4]. The solid line indicates the added region excluded by the present analysis. Asymptotic limits are indicated by the arrows. The discontinuity at $m_{\mathfrak{q}}=m_{\mathfrak{g}}$ reflects the change in the expected decay chain. Squark masses below 45 $\mathrm{GeV} / \mathrm{c}^{2}$ are excluded by data from LEP [10]. 


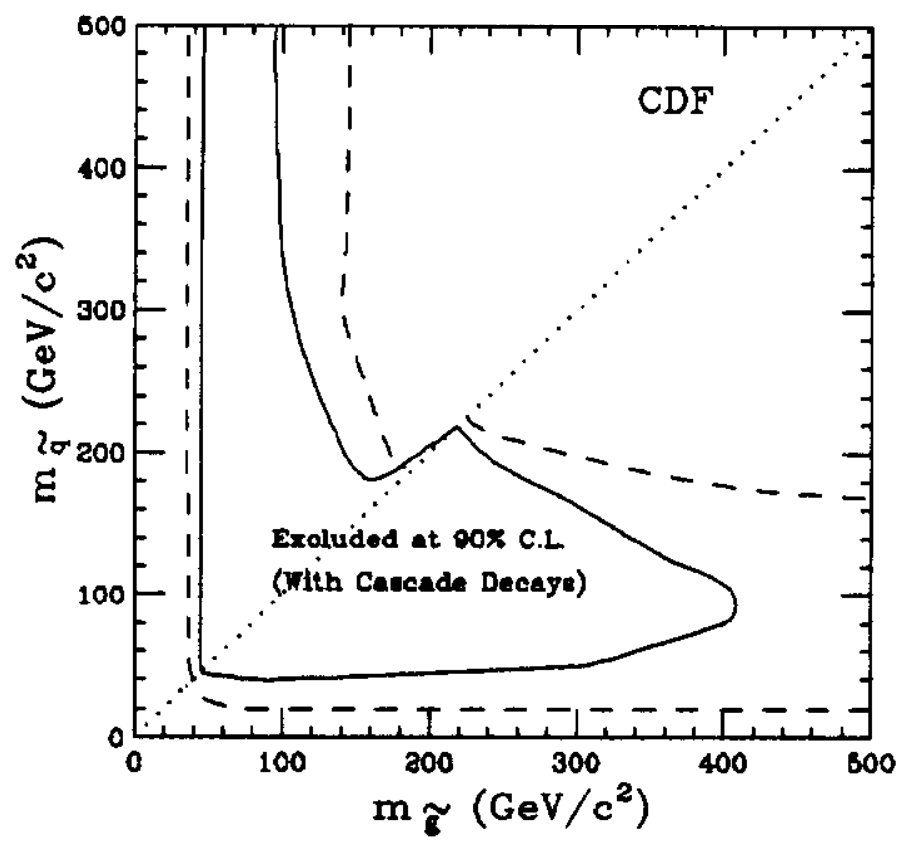

Figure 7: The solid line enclosed region of squark and gluino masses is excluded at $90 \%$ C.L for a version of SUSY with cascade decays, $\mu=-250 \mathrm{GeV}$, $\tan \beta=2$, and $m_{H}=500 \mathrm{GeV} / \mathrm{c}^{2}$. For comparison, the dashed line shows the limits corresponding to no cascade decays. 
[6] G. Marchesini, B. R. Webber, Nucl. Phys. B238, 1 (1984); B. R. Webber, Nucl. Phys. B238, 492 (1984); Marchesini and Webber, Nucl. Phys. B310, 461 (1988).

[7] F. A. Berends, W. T. Giele, H. Knijf, R. Kleiss and W. J. Stirling Phys. Lett. B224, 237 (1989).

[8] F. Paige and S. Protopopescu, BNL Report No. BNL 38034, 1986 (unpublished). We used ISAJET version 6.22.

[9] H. Baer et al., Phys. Rev. D44, 207 (1991).

[10] DELPHI Collaboration, P. Abreu et al., Phys. Lett. B247, 148 (1990). 